\title{
THE COVARIATION FUNCTION FOR SYMMETRIC $\alpha$-STABLE RANDOM VARIABLES WITH FINITE FIRST MOMENTS
}

\author{
Dedi Rosadi
}

\begin{abstract}
In this paper, we discuss a generalized dependence measure which is designed to measure dependence of two symmetric $\alpha$-stable random variables with finite mean $(1<\alpha \leq 2)$ and contains the covariance function as the special case (when $\alpha=2)$. We shortly discuss some basic properties of the function and consider several methods to estimate the function and further investigate the numerical properties of the estimator using the simulated data. We show how to apply this function to measure dependence of some stock returns on the composite index LQ45 in Indonesia Stock Exchange.
\end{abstract}

\section{INTRODUCTION}

It has been known that many popular models in finance have been developed under assumption that the returns distribution is multivariate normal. However, from numerous empirical studies (see e.g., Rydberg [14]; Rachev and Mittnik, [10]; Rosadi, [13]), the normality assumption for many empirical asset returns data can not be justified. It has been shown that many asset returns are typically leptokurtic (heavy-tailed and peaked around the center). In other words, it is found empirically that the probability that extreme events can happen, larger than the normal distribution can explain.

The class of stable distributions, of which the normal distribution is a special case, represents a natural generalization of the Gaussian distribution, and provides a popular alternative for modeling leptokurtic data. In many empirical studies

Received 15-09-2009, Accepted 21-11-2009.

2000 Mathematics Subject Classification: $37 \mathrm{M} 10$

Key words and Phrases: covariation function, infinite variance, FLOM estimator 
(see e.g., Rachev and Mittnik, [10]; Rosadi, [13]), it has been shown that the nonGaussian stable distributions with parameter index of stability $1<\alpha<2$ are more appropriate for modeling asset returns, while preserving the desirable properties of the normal. When the index $\alpha$ is less than $2(\alpha<2)$, the second moments of the stable distribution are infinite, therefore, the dependence between two random variables can not be described using covariance. When the second moments do not exist, several dependence measures has been proposed in literature, such as the covariation function, the codifference function and the dynamical function (see Rosadi, [12]). In this paper, we focus our discussion on the covariation function.

The rest of this paper is organized as follows. In the next section we shortly overview several concepts related to the stable distributions which will be necessary for the continuation of this paper. In section three, we discuss the covariation function and the estimator for the covariation function. In last section, we investigate the numerical properties of this estimator using the simulated data, and show the practical application of the covariation function to measure dependence of some stock returns on the composite index LQ45 in Indonesia Stock Exchange.

\section{INTRODUCTION TO STABLE LAWS 2.1. UNIVARIATE STABLE DISTRIBUTIONS}

The random variable $X$ is said to be (univariate)-stable if for any positive number $a$ and $b$, there is a positive number $c$ and real number $d$ such that

$$
a X_{1}+b X_{2} \stackrel{d}{=} c X+d
$$

where $X_{1}$ and $X_{2}$ denote independent copies of $X$, and $\stackrel{d}{=}$ denotes equality in distribution. The stable random variable $X$ is often parameterized using their characteristics function (for $\theta \in \mathrm{R}$ )

$\Phi_{X}(\theta)=E(\exp (i \theta X))=\exp \left\{-\sigma^{\alpha}|\theta|^{\alpha}\left(1-i \beta \operatorname{sign}(\theta) \tan \frac{\pi \alpha}{2}\right)+i \mu \theta\right\}$, untuk $\alpha \neq 1$

and

$$
\Phi_{X}(\theta)=\exp \left\{-\sigma|\theta|\left(1+i \beta \frac{2}{\pi} \operatorname{sign}(\theta) \ln |\theta|\right)+i \mu \theta\right\}, \text { untuk } \alpha=1
$$

There are four parameters to describe the univariate laws: $\alpha, \beta, \sigma$ and $\mu$. Here $\alpha$ $(0<\alpha \leq 2)$ is the index of stability; $\beta(-1 \leq \beta \leq 1)$ is the skewness; $\sigma(\sigma \geq 0)$ is the scale parameter and $\mu \in \mathrm{R}$ is the location parameter. If $\alpha=2, X$ is $\mathrm{N}\left(\mu, 2 \sigma^{2}\right)$ distributed. When $\alpha$ is smaller, $X$ is more leptokurtic. When $\alpha<2$, the absolute moment of the order $p$ if finite only for $p<\alpha$. For financial data, only $\alpha>1$ is found in practice, therefore in this paper, we assume $\alpha>1$. When $\beta=0$ and $\mu=0, X$ is called symmetric alpha-stable $(\mathrm{S} \alpha \mathrm{S})$ where $\Phi_{X}(\theta)=\exp \left(-\sigma^{\alpha}|\theta|^{\alpha}\right)$. Stable random variables have continuous probability density functions (Zolotarev, [16]), however, except for a few cases, it cannot be written in an explicit form. 


\subsection{MULTIVARIATE STABLE DISTRIBUTIONS}

A random vector $\mathbf{X}=\left(X_{1}, X_{2}, \cdots, X_{n}\right)$ is said to be a stable random vector in $\mathrm{R}^{n}$ if for any positive numbers $a$ and $b$, there is a positive number $c$ and a vector d such that

$$
a \mathbf{X}_{1}+b \mathbf{X}_{2} \stackrel{d}{=} c \mathbf{X}+d
$$

where $\mathbf{X}_{1}$ and $\mathbf{X}_{2}$ are independent copies of $\mathbf{X}$. Let $\mathbf{X}=\left(X_{1}, X_{2}, \cdots, X_{n}\right)$ be a stable random vector. Then any linear combination of the components of $\mathbf{X}$ of the type $Y=\sum_{k=1}^{n} b_{k} X_{k}$ is one dimensional $\alpha$-stable with the same index $\alpha$ for every $\mathbf{b}=\left(b_{1}, b_{2}, \cdots, b_{n}\right)$. On the other hand, let $\mathbf{X}=\left(X_{1}, X_{2}, \cdots, X_{n}\right)$ be a random vector in $\mathrm{R}^{n}$ and suppose any linear combination $Y=\sum_{k=1}^{n} b_{k} X_{k}$ is one dimensional stable. If $\alpha>1$ then $\mathbf{X}$ is stable random vector in $\mathrm{R}^{n}$.

The vector random stable $\mathbf{X}=\left(X_{1}, X_{2}, \cdots, X_{n}\right)$ with dimension $n$ is a symmetric $\alpha$-stable random variable (jointly $S \alpha S$ ), and has the characteristic function (for $\alpha>1$ )

$$
\Phi_{\mathbf{X}}(\theta)=\Phi((\theta, \mathbf{X}))=E\left(\exp \left(i \sum_{k=1}^{n} \theta_{k} X_{k}\right)\right)=\exp \left\{-\int_{\mathbf{S}_{n}}|(\theta, \mathbf{s})|^{\alpha} \Gamma(d s)\right\}
$$

where $\Gamma$ is unique symmetric finite measure (called spectral measure) which is defined on a unit sphere $\mathbf{S}_{n}=\left\{\mathbf{s}=\left(s_{1}, s_{2}, \cdots, s_{n}\right) \mid\|\mathbf{s}\|=1\right\}$. If $\mathbf{X}$ is a symmetric random variable, then the spectral measure is symmetric and we obtain

$$
\Phi_{\mathbf{X}}(\theta)=\exp \left\{-\int_{\mathbf{S}_{n}}|(\theta, \mathbf{s})|^{\alpha} \Gamma(d s)\right\}
$$

The scale parameter of the linear combination of the components of vector $\mathbf{X}$ is given as

$$
v_{\alpha}((\theta, \mathbf{X}))=\sigma^{\alpha}(\theta)=\int_{S_{n}}|(\theta, \mathbf{s})|^{\alpha} \Gamma(d s)
$$

More information on stable laws can be found in e.g., Samorodnitsky and Taqqu $([15])$.

\section{THE COVARIATION FUNCTION}

It has been known that the covariance function can completely describe the dependence structure from vector random normally distributed. However, when $\alpha<2$, the covariance function is not defined. In the following, we described the covariation function as a generalization of the covariance function for $\mathrm{S} \alpha \mathrm{S}$ process with $1<\alpha \leq 2$. 
Definition 4.1. (Samorodnitsky and Taqqu, [15]) Let $X_{1}$ and $X_{2}$ be jointly $S \alpha S$ with $\alpha>1$ and let $\Gamma$ be the spectral measure of the random vector $\left(X_{1}, X_{2}\right)$. The covariation of $X_{1}$ on $X_{2}$ is the real number

$$
\left[X_{1}, X_{2}\right]_{\alpha}=\int_{S_{2}} s_{1} s_{2}^{<\alpha-1>} \Gamma(d s)
$$

where $a^{<\alpha-1>}=|a|^{\alpha-1} \operatorname{sign}(a)$.

An alternative definition for the covariation function is given in Samorodnitsky and Taqqu ([15]), Definition 2.7.3. The properties of the covariation function can be listed below (see Samorodnitsky and Taqqu, [15] for complete proofs):

- $\left[X_{1}, X_{2}\right]_{2}=\frac{1}{2} \operatorname{Cov}\left(X_{1}, X_{2}\right)$

- In general, the covariation function is not symmetric in its argument, $\left[X_{1}, X_{2}\right]_{\alpha} \neq$ $\left[X_{2}, X_{1}\right]_{\alpha}$

- $\left[a X_{1}, b X_{2}\right]_{\alpha}=a b^{<\alpha-1>}\left[X_{1}, X_{2}\right]_{\alpha}$, for all $a, b \in \mathrm{R}$

- The covariation function is additive in the first argument. Let $\left(X_{1}, X_{2}, X_{3}\right)$ be jointly $\mathrm{S} \alpha \mathrm{S}$. Then for $a, b \in \mathrm{R}$

$$
\left[a X_{1}+b X_{2}, X_{3}\right]_{\alpha}=a\left[X_{1}, X_{3}\right]_{\alpha}+b\left[X_{2}, X_{3}\right]_{\alpha}
$$

However, in general it is not linear in the second argument,

$$
\left[X_{1}, X_{2}+X_{3}\right]_{\alpha} \neq\left[X_{1}, X_{2}\right]_{\alpha}+\left[X_{1}, X_{3}\right]_{\alpha}
$$

- If $X_{1}$ and $X_{2}$ are jointly $\mathrm{S} \alpha \mathrm{S}$ and independent, then $\left[X_{1}, X_{2}\right]_{\alpha}=0$

Furthermore, let $X_{1}$ and $X_{2}$ are jointly $\mathrm{S} \alpha \mathrm{S}$ with $1<\alpha \leq 2$, we can define the covariation coefficient $X_{1}$ on $X_{2}$ (see also Nikias and Shao, [7])

$$
\lambda=\frac{\left[X_{1}, X_{2}\right]_{\alpha}}{\left[X_{2}, X_{2}\right]_{\alpha}} \text { or } \lambda=\frac{\left[X_{1}, X_{2}\right]_{\alpha}}{\left[X_{2}\right]_{\alpha}^{\alpha}}
$$

We also have that for $X_{1}$ and $X_{2}$ are jointly S $\alpha$ S with $1<\alpha \leq 2, E\left(X_{1} \mid X_{2}\right)=\lambda X_{2}$ (Samorodnitsky and Taqqu, [15], Theorem 4.1.2.)

The above definition of the covariation function depends on the spectral measure, therefore, it makes the function less practical application. However, we can describe its relation with Fractional Lower Order Moment (FLOM) as follows (Cambanis and Miller, [3]): given $X_{1}$ and $X_{2}$ are jointly $\mathrm{S} \alpha \mathrm{S}$ and $1<\alpha \leq 2$. Let us denote the dispersion of $X_{2}$ as $\sigma_{X_{2}}^{\alpha}=\left[X_{2}\right]_{\alpha}^{\alpha}=\left[X_{2}, X_{2}\right]_{\alpha}$, therefore

$$
\lambda_{X_{1}}, X_{2}=\frac{E\left(X_{1} X_{2}^{<p-1>}\right)}{E\left(\left|X_{2}\right|^{p}\right)}, 1 \leq p<\alpha
$$


and

$$
\left[X_{1}, X_{2}\right]_{\alpha}=\frac{E\left(X_{1} X_{2}^{<p-1>}\right)}{E\left(\left|X_{2}\right|^{p}\right)} \sigma_{X_{2}}^{\alpha}, 1 \leq p<\alpha
$$

Furthermore, if we consider $U_{i}, i=1,2, \ldots, n$ is a sequence of independent random variabels $\mathrm{S} \alpha \mathrm{S}$ with dispersion $\sigma_{i}$. For any real numbers $a_{1}, \cdots, a_{n}$ and $b_{1}, \cdots, b_{n}$, where not all $b_{i}$ is equal to zero, define $X=a_{1} U_{1}+\cdots+a_{n} U_{n}$ and $Y=b_{1} U_{1}+$ $\cdots+b_{n} U_{n}$. We obtain (see e.g., Nikias and Shao, [7])

$$
\left[X_{1}, X_{2}\right]_{\alpha}=\sigma_{1} a_{1} b_{1}^{<\alpha-1>}+\cdots+\sigma_{n} a_{n} b_{n}^{<\alpha-1>}
$$

and

$$
\lambda_{X_{1}, X_{2}}=\frac{\sigma_{1} a_{1} b_{1}^{<\alpha-1>}+\cdots+\sigma_{n} a_{n} b_{n}^{<\alpha-1>}}{\sigma_{1}\left|b_{1}\right|^{\alpha}+\cdots+\sigma_{n}\left|b_{n}\right|^{\alpha}}
$$

\section{THE ESTIMATOR OF COVARIATION FUNCTION}

Several estimator for covariation function have been considered in the literature, such as Best Linear Unbiased Estimator/BLUE (Blattberg and Sargent, [2]), Least Square method (Kanter and Steiger, [6]), Fractional Lower Order Moments/FLOM estimator (see e.g., Nikias and Shao, [7]), Cheng-Rachev's method (Cheng and Rachev, [5]) and Screened Ratio estimator (Kanter and Steiger, [6]). Below we only describe the FLOM method, Screened Ratio and Least Square estimator.

Given independent samples $\left(X_{1}, Y_{1}\right), \cdots,\left(X_{n}, Y_{n}\right)$. The FLOM estimator for $\lambda_{X Y}$ is defined as (Nikias and Shao, [7]).

$$
\hat{\lambda}_{F L O M(p)}=\frac{\sum_{\mathrm{i}=1}^{n} X_{i}\left|Y_{i}\right|^{p-1} \operatorname{sign}\left(Y_{i}\right)}{\sum_{\mathrm{i}=1}^{n}\left|Y_{i}\right|^{p}}
$$

for $1 \leq p<\alpha$. This estimator is relatively simple, however it is known that the optimal values of $p$ is unknown.

The Screened Ratio Estimator is defined as

$$
\hat{\lambda}_{S C R}=\sum_{i=1}^{n}\left(X_{i} Y_{i}^{-1} \chi_{Y_{i}}\right) / \sum_{i=1}^{n}\left(\chi_{Y_{i}}\right)
$$

where $\chi_{Y_{i}}=1$ if $Y_{i}>0$ and 0 for the other case. The Least Square estimator is obtained by minimizing the error $\sum_{i=1}^{n}\left(X_{i}-\lambda Y_{i}\right)^{2}$. We obtain

$$
\hat{\lambda}_{L S}=\sum_{i=1}^{n}\left(X_{i} Y_{i}^{-1}\right) / \sum_{i=1}^{n}\left(Y_{i}^{2}\right)
$$


This estimator is inconsistent estimator in the infinite variance, and we consider it here only for the comparative purpose.

\section{SIMULATION STUDIES}

In this section, we investigate the numerical properties of the estimator of the covariation function. Using $\mathrm{R}$ version 2.7.2 ( $\mathrm{R}$ Development Core Team, [11]), we generate two independent random samples $U_{1}$ and $U_{2}$ using the algorithm presented in Chambers, Mallow and Stuck ([4]) for several values of $\alpha$ and sample size 5000 where the replication is taken to be 50 times. We further define the random variable $X=a_{1} U_{1}+a_{2} U_{2}$ and $Y=b_{1} U_{1}+b_{2} U_{2}$. For this simulation study, we use $a_{1}=0.75, a_{2}=0.25, b_{1}=0.18$ and $b_{2}=0.78$, therefore, we obtain the true covariation coefficient of $\mathrm{X}$ on $\mathrm{Y}$ is equal to $\lambda=$ $\left(0.75 *(0.18)^{\alpha-1}+0.25 *(0.78)^{\alpha-1}\right) /\left((0.18)^{\alpha}+(0.78)^{\alpha}\right)$. The covariation of $\mathrm{X}$ on $\mathrm{Y}$ can be obtained by multiplying $\lambda$ with $\left((0.18)^{\alpha}+(0.78)^{\alpha}\right)$. The covariation coefficient is estimated using Least Square (LS) method, Screened Ratio Estimator (SCR) and FLOM (we consider two cases, $p=1$ and $p<\alpha$. Here, we use $p=1.05$ when $\alpha=1.1$ and $p=1.1$ for the other $\alpha \mathrm{s}$ ). The simulation results are summarized in figure 1. The findings show that in general the FLOM estimator with $p=1$ perform the best, it is an accurate estimator for the covariation coefficient when $\alpha$ is getting closer to $2(\alpha>1.5)$ although it is become more volatile when $\alpha$ is closer to 1 .

\section{EMPIRICAL RESULTS}

To illustrate the usability of the method discussed above, we use the stocks chosen from LQ45 index listed in Indonesia Stock Exchange. For this purpose, we collect some daily closing price data from 6 stocks during the period Januari 1, 2003 until Desember 31, 2007. The data is transformed into daily returns using $Y_{t}=\ln \left(X_{t+1} / X_{t}\right), t=1, \cdots, N-1$.

For checking the possibility of using process with infinite second moment, we use the diagnostic method as discussed in Rosadi ([13]). The examples of diagnostic plot for daily and weekly data (as an average of five days data) for the Indosat Tbk. 's stock returns are given in Figure 2 and 3 . The converging variance plot and the plot of ratios between the maximum and partial sum indicate that the second moments of the process are infinite. Based on the density plot, the nonnormal stable distributions show a better shape to the data than compared to the fitting result using the normal distribution (where the sample mean and the sample variance are used as the estimated parameters). The similar results can also be obtained for the other stocks considered in this study. We note that for producing this figure, we estimate the parameter of stable distribution using the numerical maximum likelihood method as described in Nolan $([8])$, implemented in package fBasics in Rmetrics. 
alpha=1.1

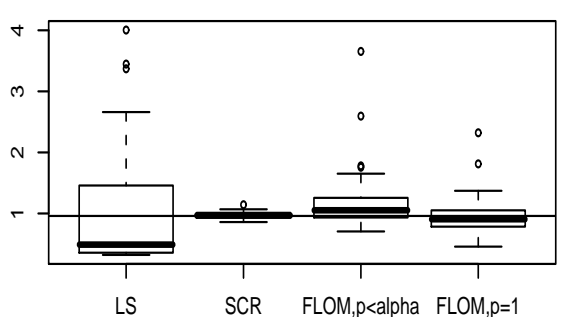

alpha $=1.5$

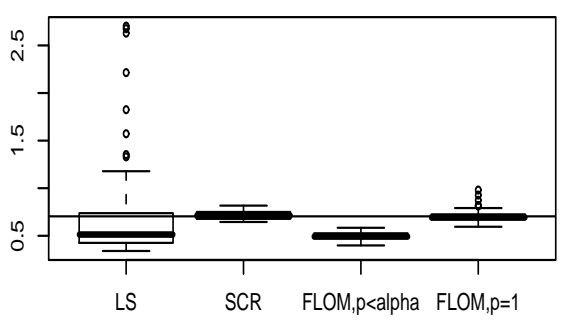

alpha $=2.0$

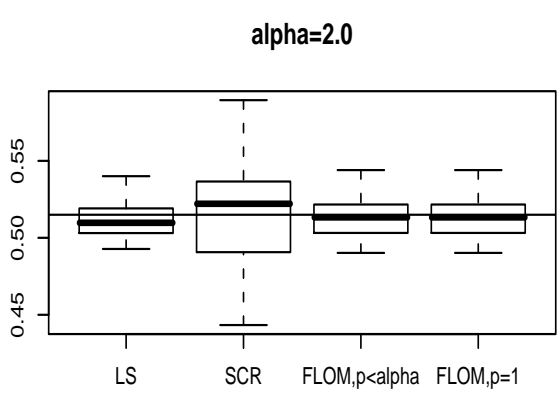

alpha $=1.3$

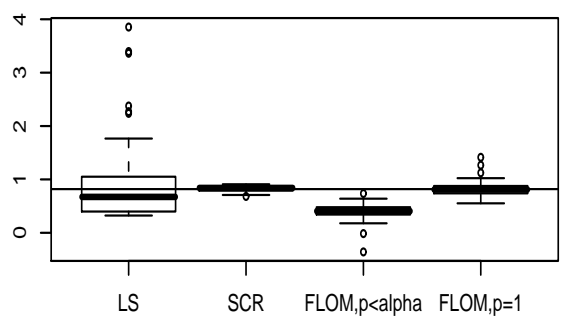

alpha $=1.9$

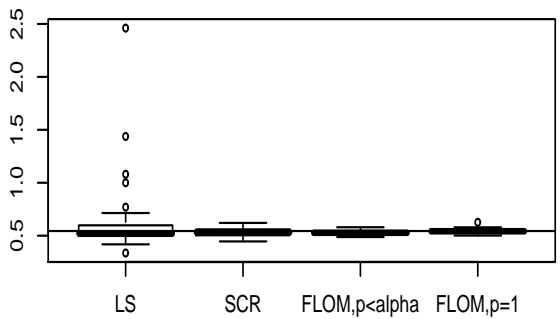

Figure 1: The accuracy of the estimator of covariation function for several values of $\alpha$. The straight lines denote the true values of the covariation function in each cases. 

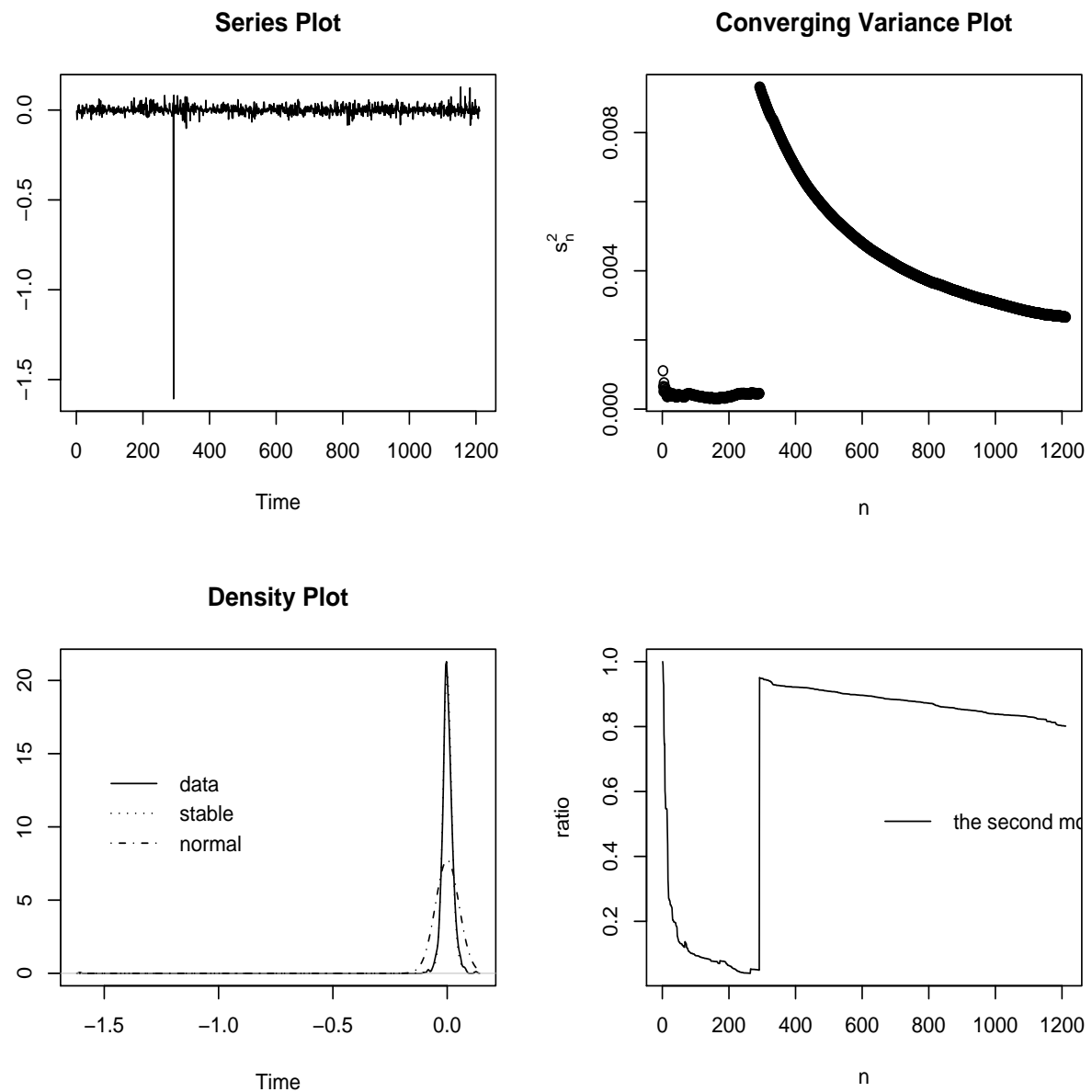

Figure 2: Diagnostic plot for daily Indosat Tbk. 's returns, sample size N=1121 
Series Plot

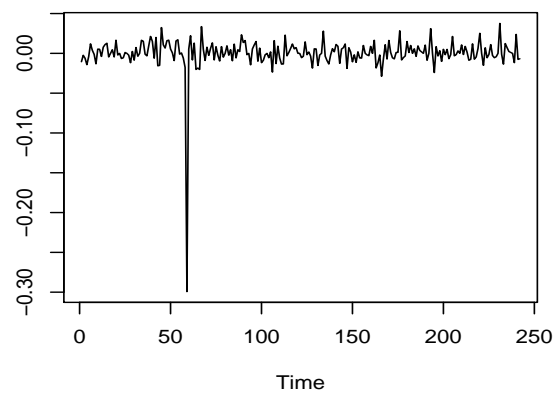

Density Plot

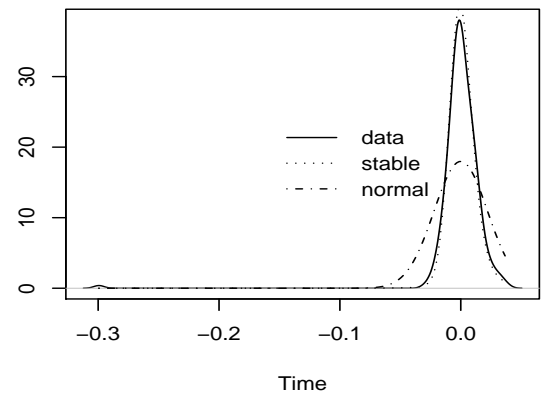

Converging Variance Plot
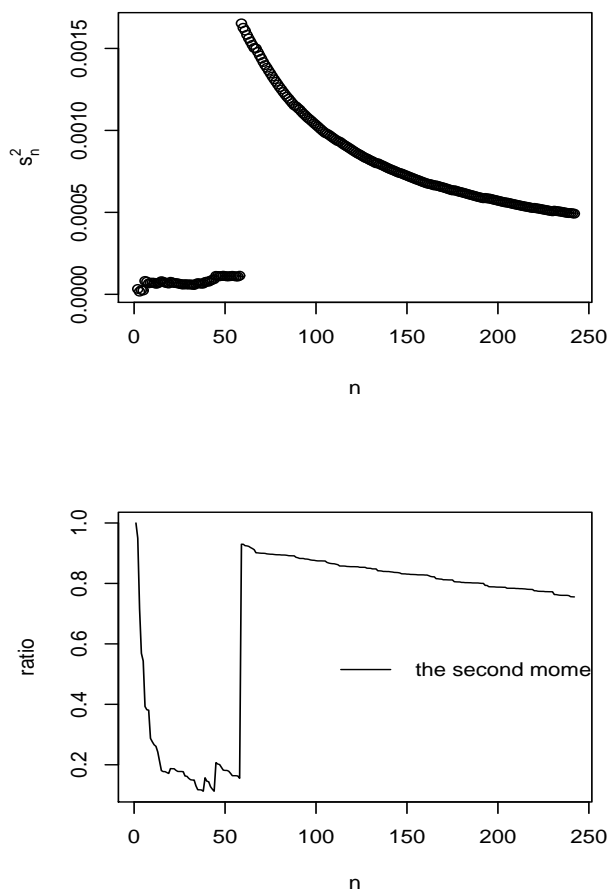

Figure 3: Diagnostic plot for weekly Indosat Tbk.'s returns, sample size N=224 
Although the second moments of the processes that generated the data might be infinite, they are not necessary stable. To check the stability of data, one should check the estimates of the index $\alpha$ for the data over different time horizons (e.g., over daily, weekly and monthly horizons. See for instance Paolella, [9]). This verification can confirm that the (necessarily i.i.d.) data could have been generated from a stable law, because this property truly characterizes the stability of the sum of any finite number of i.i.d. stable random variables. If the true distribution of the data is a stable law, the estimated tail indexes of summed non-overlapping $j$ - length segments of the data can be expected roughly constant as a function of $j, j=1,2, \cdots$, while for non stable data, the estimates should tend to increase towards two as j increases.

For checking the summability property of the data, we estimate the parameter of stable distribution using the Nolan's numerical maximum likelihood method, implemented in package fBasics in Rmetrics. The time horizons uses in the study are $j=1,2, \cdots, 10$, where for $j>1$, we calculate the average of the data over nonoverlapping $j$-length segments of the data. Our choice of maximum time horizon $j=10$ days here is only restricted by the length of the data available in the study. The fitting results using non-normal stable distribution for the stocks returns are given in the following table.

Inspection of the estimated values of the characteristic exponent $\alpha$ ranges from 1.45 to 1.95 , with an average is approximately $\hat{\alpha}=1.7$. This value is close to the value found by several authors in different market (see e.g. Belkacem, Levy and Vehel, [1]).

As an illustration for the application of the result presented in the previous sections, in what follows, we calculate the covariation coefficient $\lambda$ between the stocks and the index LQ45. It has been shown in Belkacem et. al. ([1]) that the the covariation coefficient $\lambda$ between the stock and the market index can be interpreted as the $\beta_{i}$ in the stable CAPM model. Under certain conditions, Belkacem et. al. ([1]) shows that the "stable" CAPM generalizes the Gaussian CAPM, it has the following form

$$
E\left(R_{i}\right)-R_{f}=\beta_{i}\left(E\left(R_{m}\right)-R_{f}\right)
$$

where

$$
\beta_{i}=\frac{\left[R_{i}, R_{m}\right]_{\alpha}}{\left\|R_{m}\right\|_{\alpha}^{\alpha}}
$$

The "stable" CAPM presents the generalized equilibrium relationship between risk and return for a given security. The return over the risk free rate $E\left(R_{i}\right)-R_{f}$ is called the risk premium for a security i. $E\left(R_{m}\right)-R_{f}$ is the price of the risk. $\beta_{i}$ is "generalized beta coefficient" which measures the volatility of the security's rate of return relative to changes in the market's rate of return, and can be interpreted as the quantity of risk, in the similar way as in the classical case. The estimated value of $\beta_{i}$ for daily returns using index LQ45 as the "proxy" of market portfolio is summarized in Table 2. It shows that there are three responsive assets (INDF, INTP, TLKM) and the others are less sensitive to the change of market portfolio. 
Table 1. Estimated of $\alpha$-values for samples defined with respect to the time intervals of different sizes. The averages denotes the mean and standard deviation of $\hat{\alpha}$ over all time frequencies considered here

\begin{tabular}{|c|c|c|c|c|c|c|c|c|c|c|c|}
\hline $\begin{array}{l}\text { Frequency } \\
\text { (days) }\end{array}$ & 1 & 2 & 3 & 4 & 5 & 6 & 7 & 8 & 9 & 10 & \multirow{2}{*}{ Average } \\
\hline Sample Size & 1211 & 605 & 403 & 302 & 242 & 201 & 173 & 151 & 134 & 121 & \\
\hline \multicolumn{11}{|l|}{ Stocks } & \\
\hline Index LQ45 & 1.76 & 1.58 & 1.53 & 1.53 & 1.68 & 1.86 & 1.81 & 1.80 & 1.61 & 1.77 & $1.69 \pm 0.12$ \\
\hline $\begin{array}{l}\text { IHSG Com- } \\
\text { posite Index }\end{array}$ & 1.62 & 1.56 & 1.57 & 1.49 & 1.61 & 1.72 & 1.80 & 1.87 & 1.75 & 1.80 & $1.68 \pm 0.13$ \\
\hline $\begin{array}{ll}\text { Indosat } & \text { Tbk } \\
\text { (ISAT) } & \end{array}$ & 1.73 & 1.76 & 1.77 & 1.78 & 1.72 & 1.77 & 1.65 & 1.59 & 1.71 & 1.78 & $1.73 \pm 0.06$ \\
\hline $\begin{array}{l}\text { Bank Central } \\
\text { Asia (BBCA) }\end{array}$ & 1.83 & 1.85 & 1.80 & 1.75 & 1.62 & 1.56 & 1.90 & 1.91 & 1.69 & 1.72 & $1.76 \pm 0.12$ \\
\hline $\begin{array}{ll}\text { Astra } & \text { Agro } \\
\text { Lestari } & \\
\text { (AALI) } & \end{array}$ & 1.63 & 1.65 & 1.85 & 1.78 & 1.93 & 1.78 & 1.95 & 1.47 & 1.87 & 1.72 & $1.76 \pm 0.15$ \\
\hline $\begin{array}{l}\text { Indofood Suk- } \\
\text { ses (INDF) }\end{array}$ & 1.74 & 1.78 & 1.70 & 1.68 & 1.80 & 1.64 & 1.73 & 1.77 & 1.68 & 1.70 & $1.72 \pm 0.05$ \\
\hline $\begin{array}{l}\text { Indocement } \\
\text { (INTP) }\end{array}$ & 1.65 & 1.58 & 1.64 & 1.97 & 1.73 & 1.55 & 1.57 & 1.71 & 1.65 & 1.46 & $1.65 \pm 0.14$ \\
\hline $\begin{array}{l}\text { Telekomunikasi } \\
\text { Indonesia } \\
\text { (TLKM) }\end{array}$ & 1.79 & 1.77 & 1.70 & 1.85 & 1.84 & 1.77 & 1.87 & 1.95 & 1.52 & 1.89 & $1.80 \pm 0.12$ \\
\hline
\end{tabular}

Table 2. Estimated values of covariation coefficient between stocks and market returns

\begin{tabular}{|l|l|}
\hline Stocks & $\hat{\lambda}(\text { Stock }, L Q 45)_{\alpha=1.7}$ \\
\hline Indosat Tbk (ISAT) & 0.80 \\
\hline Bank Central Asia (BBCA) & 0.89 \\
\hline Astra Agro Lestari (AALI) & 0.82 \\
\hline Indofood Sukses (INDF) & 1.02 \\
\hline Indocement (INTP) & 1.05 \\
\hline Telekomunikasi Indonesia (TLKM) & 1.23 \\
\hline
\end{tabular}


Acknowledgement. The research support from DIKTI Indonesia via Fundamental Research Project number UGM/PID/583/2007 is gratefully acknowledged.

\section{REFERENCES}

1. L. Belkacem, J. L. V'Ehel And C. WAlter, "CAPM, risk and portfolio selection in stable markets", Fractals , 8(1) (2000), 99-115.

2. R. Blattberg, and T. Sargent, "Regression with Non-Gaussian Stable Disturbances: some sampling results", Econometrica, 39 (3) (1971), 501-510.

3. S. Cambanis and G. Miller, "Linear problems in p-th order and Stable Processes", SIAM J.Appl. Math., 41 (1981), 43-69.

4. J.M. Chambers, C. L. Mallows and B.W. Stuck, "A method for simulating stable random variables", Journal of the American Statistical Association, 71 (1976), 340-344.

5. B.N. Cheng and S.T. Rachev, "Multivariate Stable Futures Prices", Journal of the Mathematical Finance, 5 (1995), 133-153.

6. M. Kanter And W.L. Steiger, "Regression and Autoregression with Infinite Variance", Advanced Applied Probability, 6 (1974), 768-783.

7. C.L. Nikias and M. Shao, Finite-Dimensional Vector Spaces, Wiley, New York, 1995.

8. J.P. Nolan, "Numerical Calculation of Stable Densities and Distribution Functions", Preprint, University Washington DC, 16 pages, 1999

9. M.S. PaOlella , "Testing the stable Paretian assumption". Mathematical and Computer Modelling, 34 (2001), 1095-1112.

10. S.T. Rachev and S. Mittnik, Stable Paretian Models in Finance, Wiley, Chichester, 2000.

11. R Development Core Team, $R$ : A language and environment for statistical computing, R Foundation for Statistical Computing, Vienna, Austria. ISBN 3-900051-00-3, 2008.

12. D. RosADI, "Asymptotic behavior of the codifference and the dynamical function for ARMA models with Infinite variance", Journal of Indonesian Mathematical Society (MIHMI) , 11 (1) (2005a), 59-69.

13. D. Rosadi, "Data fitting and Goodness of Fit test for Stable distribution and Its application in financial modeling". Proceedings of National Mathematics and Science Conference, Faculty of Mathematics and Science, Gadjah Mada University, (2005b). In Bahasa Indonesia.

14. T.H. Rydberg, "Realistic Statistical Modeling of Financial data ", Proceedings of 51th International Statistical Institute Conference, Istambul, 1997.

15. G. SAmorodnitsky And M.S. TAQQU, Stable Non-Gaussian Processes: Stochastic Models with Infinite Variance, Chapman and Hall, New York, 1994.

16. V.M. Zolotarev, One Dimensional Stable Distributions, American Mathematical Society, Providence, RI, 1986.

D. Rosadi: Department of Mathematics, Gadjah Mada University, Indonesia.

E-mail: dedirosadi@ugm.ac.id 